\section{EL PROBLEMA DE LA CAZA VISTO POR UN GENETISTA}

\author{
José Antonio Dávila García \\ Consejo Superior de Investigaciones Científicas - Universidad de \\ Castilla-La Mancha - Junta de Comunidades de Castilla-La Mancha \\ ORCID iD: http://orcid.org/0000-0002-8755-8589 \\ joseantonio.davila@uclm.es
}

Cómo citar este artículo/Citation: Dávila García, J. A. (2017). El problema de la caza visto por un genetista. Arbor, 193 (786): a416. doi: http://dx.doi.org/10.3989/arbor.2017.786n4005

Recibido: 30 abril 2015. Aceptado: 04 febrero 2016.

RESUMEN: La caza tiene problemas para dar y tomar. Produce y sufre problemas de todo tipo, aunque solo hablaré de los genéticos. La caza está en declive sobre todo porque a la naturaleza le va mal. Sin embargo, la caza no solo es fuente de problemas, sino también de soluciones. Hay que compatibilizar caza y conservación de la naturaleza de modo que se haga un uso sostenible de la fauna y los ecosistemas. Hay conocimientos científicos y técnicos suficientes para conseguirlo. Quizá lo que no hay es voluntad. ¿Cómo les cuento de la caza si de la caza ya se ha dicho todo? Lo único que se me ocurre es dar una visión muy personal con palabras sencillas. Espero contar algo nuevo, aunque me consta el desinterés manifiesto de algunas personas por que se les cuenten historias que les hagan pensar de otra manera o siquiera dudar.

PALABRAS CLAVE: Caza; conservación; genética.

\section{THE PROBLEM OF HUNTING AS SEEN BY A GENETICIST}

Copyright: () 2017 CSIC. Este es un artículo de acceso abierto distribuido bajo los términos de la licencia Creative Commons Attribution (CC BY) España 3.0.

ABSTRACT: There are many problems to choose from as regards hunting. It either generates or suffers from all sorts of problems, although here I will only refer to genetic ones. Hunting is declining mainly because nature is not doing so well. However, hunting is not only a source of problems, but also of solutions. We must reconcile hunting and nature conservation, to permit a sustainable use of wildlife and ecosystems. There is already sufficient scientific and technical knowledge to achieve this, but perhaps not the will to do so. How can I tell you about hunting if everything has already been said? All that occurs to me is to give a very personal view using simple words. I wish I could say something new, but I am well aware of some people's clear disinterest in listening to stories that make them think differently, or perhaps question their previous knowledge.

KEYWORDS: Hunting; conservation; genetics. 
"Para empezar por el principio, diré que la perdiz roja es un pájaro que todavía está ahí. Los pájaros de ellos, los pájaros de los otros, son, a menudo, pájaros que les han puesto. Parece natural que con alguna ventaja habíamos de contar los pueblos subdesarrollados. Con esto se da por supuesto que la civilización opera contra la caza, o, todo sea dicho con palabras pobres, que el tractor y la cosechadora se comen la perdiz".

Miguel Delibes. El libro de la caza menor (1964).

\section{PONIENDO EL PROBLEMA EN PERSPECTIVA}

La caza está en declive porque para cazar hay que irse al campo. El declive de la caza es simultáneo a la pérdida de paisajes y especies. La naturaleza está sufriendo una crisis de extinciones de especies como nunca ha ocurrido en la historia del planeta. La caza consiste en matar animales y algo ha tenido que ver en las extinciones de especies, aunque a escala global tiene muy poca culpabilidad y algo que agradecerle. El "problema" de la caza son muchos problemas pero el de la pérdida de naturaleza es el principal.

Voy a intentar poner el problema en perspectiva. En el año 1800 vivían 1.000 millones de personas. En el año 2000 éramos 6.000 millones y ahora pasamos de los 7.000 millones. El crecimiento de la población asusta. Se ha estimado que en 2100 serán 11.000 millones de personas (United Nations - Department of Economic and Social Affairs. Population Division). A la vez, la producción de cereales per capita empezó a disminuir a mediados de los años 80 y todavía no ha parado de seguir cayendo, mientras que la superficie de cultivo per capita ha disminuido cerca del $20 \%$ en la última década. (Funk y Brown, 2009; FAOSTAT). Cada día que pasa tomamos más tierra, más bosques y más vida silvestre, y lo hacemos de forma más agresiva. Hay que intentar pensar en la escala. La mayoría de las especies animales y vegetales están en las selvas tropicales y la situación de casi todas las selvas tropicales es desesperada. Solo nos quedan siete millones de $\mathrm{Km}^{2}$ de selva tropical. En 1999 John Terborgh calculó que el último árbol de una selva tropical se talaría en 2045. Desgraciadamente, el ritmo al que desaparecen los bosques tropicales se ha incrementado desde 1999. El 40\% de todos los bosques lluviosos tropicales está en Brasil y esa masa verde representa un papel vital en el mantenimiento de la biodiversidad de la Tierra, la hidrología regional, el clima mundial y el ciclo del $\mathrm{CO}_{2}$. Es un record planetario. También tiene el record mundial de velocidad de destrucción absoluta de bosque, deforestando más de dos millones de hectáreas al año y aumentando el ritmo según su economía crece (Butler,
2005, 16 de noviembre; Malhi et al., 2008). Los problemas están en todos lados, no solo en los bosques tropicales. También nuestros paisajes y ecosistemas están sufriendo alteraciones drásticas. Cuantificando la pérdida de naturaleza de otro modo: el $13 \%$ de las especies de aves, la quinta parte de los mamíferos, el $5 \%$ de los peces y el $8 \%$ de las plantas terrestres están amenazados de extinción seriamente. Casi todos los animales grandes están en problemas: los felinos, las grullas, los loros, las serpientes, los cánidos, los monos antropomorfos, los elefantes, los rinocerontes... todos los animales "icónicos". En dos décadas se mató al $90 \%$ de todos los rinocerontes negros, quedan menos de 400 rinocerontes de Sumatra, es posible que queden unos 600 gorilas de montaña, el águila de Filipinas está mucho peor que la imperial ibérica, la foca monje casi se ha extinguido... Los peores son los gatos. Los gatos domésticos son quizá la causa mayor de destrucción de especies de aves tras la pérdida de hábitat. No son solo los gatos. Los perros han extinguido poblaciones silvestres de cánidos transmitiendo enfermedades. El ganado también hace lo suyo y son una especie de plaga, devastando hábitats y propagando enfermedades. La tuberculosis bovina ha pasado de las vacas a los búfalos silvestres y de estos a los leones, guepardos, kudúes y babuinos en Sudáfrica, mientras que en Norteamérica se la contagiaban a los bisontes y en España a los linces (Gortázar, Che Amat y O’Brien, 2015). La única esperanza que tiene la fauna y flora amenazadas de las islas es la exterminación de las plantas, cabras, ratas, perros, gatos y conejos que hemos introducido en ellas. Aunque desde hace mucho tiempo somos conscientes de los problemas tremendos que puede suponer introducir especies exóticas, continuamos haciéndolo sin disminuir el ritmo. Consumimos el $35 \%$ de la productividad primaria de los ecosistemas continentales y usamos el $55 \%$ del agua de los ríos, lagos y otras fuentes de agua dulce. Sin embargo, el porcentaje de tierra que está protegido es mucho menor del $5 \%$ de la superficie mundial y no llega al 0,5\% la superficie marina protegida, sea lo efectiva que sea esa protección (World Bank Open Data). Esta es, pues, la escala de la destrucción de la naturaleza.

Hay que proteger la naturaleza porque la estamos destruyendo. Cuando se trata de proteger ecosistemas, mayor superficie es mejor que menor, conectado es mejor que fragmentado y natural es mejor que manejado. Eso es obvio pero complicado de conseguir en la práctica. Desgraciadamente, en la actualidad y por lo menos en el futuro inmediato, la supervivencia de cada vez más especies tendrá que depender del cuidado de fragmentos de hábitat en forma de reservas o, peor aún, zoos, hasta 
que se pueda recuperar el hábitat perdido y las causas de extinción. Muchas especies tendrán que intentar sobrevivir en pequeñas poblaciones aisladas sin espacio para crecer. Su supervivencia depende del cuidado humano. Por si fuera poco, de una manera o de otra, toda la vida silvestre está amenazada por el cambio climático. No solo afectará a la vida silvestre, sino que el cambio climático muy probablemente afectará a las poblaciones humanas. Algunas sociedades podrán hacer frente al problema, pero son de esperar rupturas sociales, movimiento de poblaciones y explotación de más recursos, que afectarán también a la naturaleza. Para qué seguir.

Hoy en día, proteger la naturaleza significa gestionarla. Los recursos son limitados, por lo que tendremos que ponernos de acuerdo en qué especies o subespecies queremos proteger, dónde y en qué números y cuál es la mejor manera de hacerlo. A largo plazo no puede haber conflicto entre el desarrollo económico y la protección de la naturaleza. A corto plazo, hay conflictos inmensos. La palabra progreso hace tiempo que la hemos sustituido por desarrollo económico. A nadie se le ha ocurrido cómo hacerse rico con linces ibéricos, gorilas de montaña o golondrinas. Para mucha gente, el gasto en la protección de especies es un lujo que no nos podemos permitir. Al final se conseguirá. Hará falta el trabajo de miles de personas y el entendimiento de millones para conseguir el cambio, pero mientras tanto, ¿qué hacemos?, ¿cuál es la mejor manera de hacerlo?, ¿debemos evitar la extinción de todas las especies?, ¿de las subespecies?, ¿'solo de algunas? La mejor manera de conservar la naturaleza es conservar paisajes. Si queremos salvar especies, tendremos que salvar el hábitat.

\section{UNA DEFENSA DE LA CAZA}

Alrededor del $62 \%$ de la superficie nacional es terreno cinegético (Estadística anual de caza. Tablas resumen 2005-2010). No todo ese territorio tiene un uso exclusivamente cinegético, en ocasiones sí, pero en cualquier caso todo él es gestionado para cazar, en mayor o menor medida y mejor o peor hecho. Para cazar hace falta naturaleza y la caza ha trabajado para que esos lugares se mantengan y prosperen en ellos las especies cinegéticas y sus depredadores aunque los quieran controlar. Existen otras figuras de protección del territorio, como parques, reservas naturales, monumentos naturales, paisajes protegidos, Red Natura 2000 y quizá otras, y dentro de muchas de ellas se puede cazar. Esas figuras de protección del territorio son espacios protegidos aislados. La caza protege mucha más superficie que además es continua en el espacio. Está bien disponer de espacios protegidos, pero es mucho mejor la protección del espacio, que hace la caza. También la caza ha cedido partes de su superficie para convertirla en espacios protegidos, y ahí está el caso paradigmático de la Reserva Natural del Monte del Pardo, que fue un coto de la Corona y es una de las mayores extensiones de ecosistema bien conservado de Europa, a las mismas puertas de Madrid.

La caza no solo se ocupa de proteger el espacio. También protege a especies concretas. Por ejemplo, el ciervo. Esta especie se extinguió en la mitad norte de la Península Ibérica. Con fines cinegéticos, a partir de los años 50 comenzaron a repoblarse diferentes sierras del norte de España con ciervos provenientes del centro y sur peninsular, principalmente de Quintos de Mora (Toledo), creándose nuevas Reservas Nacionales de Caza. En la actualidad los ciervos se encuentran en prácticamente toda la Península y las poblaciones están aumentando, lo que seguro que agradece el lobo además de los cazadores.

Junto a todo esto, el aprovechamiento cinegético produce rentas y empleo, especialmente en ambientes rurales, complementando o sustituyendo a las rentas agrarias. El medio rural se nos despuebla y cualquier actividad económica allí es bienvenida y necesaria para cohesionar socialmente el país.

Sin embargo, la caza no solo tiene aspectos positivos. Aunque la alteración y destrucción del hábitat sea la principal causa de destrucción del planeta y de pérdida de biodiversidad, la caza tiene su parte de culpa también. En primer lugar, porque la caza actúa directamente sobre los animales matándolos. Algunas especies se están matando de más y no podemos olvidar que en la historia algunas han sido cazadas hasta su extinción. Además, la caza está siendo perjudicial para la naturaleza por métodos menos directos que pegarles tiros a más animales que los que la naturaleza puede reponer. La caza altera la naturaleza de varias formas menos evidentes. Algunas de estas formas tienen consecuencias genéticas (que luego podrán tener consecuencias demográficas) y de ellas se encarga la Genética. Así pues, antes de contar algunas de estas maneras más enrevesadas por las que la caza se fastidia a sí misma, creo conveniente explicar uno o dos conceptos de genética, con palabras simples.

\section{MANERAS EN QUE LA GENÉTICA PUEDE AYUDAR AL CONOCIMIENTO Y GESTIÓN DE LA CAZA}

Cada especie y subespecie es como es por su información genética. Cada individuo también, aunque en mayor o menor medida en el aspecto (fenotipo) 
influye además el ambiente. La información genética está contenida en una molécula que es el ADN. La molécula de ADN es capaz de hacer copias de sí misma, con lo que puede pasar entre sucesivas generaciones en los seres vivos. Esta molécula es también extraordinaria porque es capaz de contener la información que nos hace a cada ser vivo ser lo que somos. En los seres vivos que nos interesan aquí, la información genética está contenida en varias moléculas de ADN y cada una de ellas forma parte de un cromosoma. La información genética está duplicada, así que el número de cromosomas de una célula es par. Cada cromosoma de una pareja contiene información para hacer lo mismo, por ejemplo, el color del pelo, aunque puede ser que en cada lugar del ADN de ambos cromosomas (cada gen) haya variantes en la información para hacer lo mismo: una variante de un gen (alelo) en un cromosoma para teñir el pelo de un color y otra variante en su otro cromosoma para teñirlo diferente. Uno de los juegos de cromosomas proviene del padre, de un espermatozoide, y el otro juego proviene de la madre, de un óvulo. Hay una pequeña cantidad de información genética que está contenida en una molécula de ADN distinta de la que hace los cromosomas. Es el ADN mitocondrial. Las mitocondrias son orgánulos de las células que tienen su propio ADN, diferente del de los cromosomas. Los espermatozoides no transfieren ADN mitocondrial al embrión, de modo que todo el ADN mitocondrial de un individuo lo ha heredado de su madre.

La Genética estudia el modo en que el ADN se transmite entre generaciones. Cuando la Genética estudia aspectos cinegéticos no suele estudiar la herencia en individuos sino en grupos. Es decir, estudia cómo es y cómo varía el acervo genético de poblaciones. Los ciervos del Monte del Pardo, por ejemplo. La base de la Genética de Poblaciones es la ley de Hardy-Weinberg. Dice que, en una población infinita donde las generaciones no se solapan, el apareamiento es libre y al azar y donde no hay mutación, migración ni selección, la composición genética permanece en equilibrio. La "composición genética" de la población son las frecuencias de los alelos de cada gen. Si nada cambia (no hay mutación, selección...), las frecuencias de los distintos alelos permanecen constantes. Podemos establecer una analogía con los apellidos de la guía de teléfonos de una ciudad grande. Las frecuencias de los apellidos son las mismas en las guías de todos los años. Conocidas las frecuencias de los apellidos (4\% de Benítez, 15\% de Sánchez, 8\% de Pérez...) se pueden calcular las frecuencias de las parejas de apellidos en la guía de teléfonos (frecuencia de Pérez Benítez, Sánchez Pérez, y así) y además la frecuencia de cada pareja de apellidos permanece constante. Cuando la composición genética de una población cambia es porque está pasando algo que la altera. Ese "algo" es una violación de las premisas del modelo de Hardy-Weinberg: que la población es infinita, las generaciones no se solapan, el apareamiento es libre y al azar y donde no hay mutación, migración ni selección. La labor del genetista es detectar un cambio en la composición genética y averiguar el motivo, el "algo" que produce el cambio. En el modelo la población es infinita. Las poblaciones finitas pierden diversidad genética generación tras generación, pero si la población es grande, esa pérdida de alelos es tan lenta que a la escala temporal humana que nos interesa es despreciable. Tampoco varía mucho las cuentas el hecho de que las poblaciones sean solapantes, es decir, que en un mismo año puedan reproducirse en la población padres, abuelos, nietos y cualquier individuo de cualquier generación viva. Las mutaciones son sucesos muy raros y podemos despreciar también su efecto, ya que van produciendo alelos nuevos a un ritmo tan lento que no es de importancia en la escala temporal de la gestión cinegética. Los apareamientos pueden ser más o menos aleatorios en algunas especies, como los conejos, o pueden no serlo completamente en especies donde determinados genotipos tienen ventaja reproductiva y unos cuantos machos acaparan todas las hembras, como los ciervos. Con todo, cuando se observa un cambio de la composición genética de una población hay que sospechar de la migración y de la selección. Los casos descritos y posibles de causas de cambios en la composición genética y sus consecuencias son numerosos, pero para muestra vale desgranar unos cuantos. A veces una población puede perder alelos y con ello perder diversidad genética debido a una interrupción del flujo genético. Eso puede ocurrir cuando una población queda dividida, por ejemplo, por una autopista. ¿Son capaces los ciervos de cruzar una autopista, aunque sea por los pasos de fauna habilitados? Para saberlo no hay más que estudiar las frecuencias de los alelos de los ciervos a uno y otro lado. A veces el flujo genético se interrumpe porque la población ha quedado aislada, por ejemplo, con un vallado cinegético que cierra toda una finca. A partir de ese momento la diversidad genética de la población irá disminuyendo, aunque el ritmo de pérdida depende del tamaño de la población que ha quedado aislada y de su diversidad genética inicial. La pérdida de diversidad puede ser lenta, o puede no darse si el vallado es algo permeable al paso; para averiguarlo basta contar las frecuencias de los alelos en la población. Hay casos en los que rápidamente se pierde gran 
diversidad genética. Por ejemplo, cuando se funda una población por la translocación de unos pocos ejemplares de otra población, pongamos por caso las poblaciones de arruís de algunas fincas. También se pierde mucha diversidad genética en poco tiempo en otros casos de "cuello de botella", casos en los que muchos animales de una población mueren y esta se recupera o no a partir de pocos individuos. Enfermedades y caza excesiva provocan cuellos de botella. A veces las frecuencias de genotipos de una población no son las que se esperarían atendiendo a las frecuencias de los alelos y eso no es por flujo genético ni por selección, sino simplemente porque lo que se pensaba que era una población son dos. Un ejemplo de esto es una población silvestre de perdices después de soltar allí algunas otras de una granja: en ese momento no hay una población sino dos, la silvestre y la de granja. Un caso curioso de la existencia de dos poblaciones en donde se pensaba que había una se describió en el corzo (Kurt, Hartl y Voelk, 1993). En ese lugar, algunos machos acaparaban la zona de bosque y eran territoriales mientras que el resto vivía en las zonas más abiertas y eran más migratorios. Allí había dos grupos familiares, dos poblaciones, una en el bosque y otra en zonas abiertas. Esas diferencias en la preferencia de hábitat podrían deberse a adaptaciones diferentes a los dos ambientes y eran mantenidas por bajos niveles de flujo genético entre ambas poblaciones. Es interesante que, cuando la presión de la caza aumentó, se forzó de alguna manera el flujo genético entre ambos sistemas sociales y esta diferenciación entre los dos grupos de corzos empezó a desaparecer. También la selección, natural o artificial, cambia las frecuencias de algunos alelos. Por ejemplo, no sería de extrañar que las frecuencias de determinados alelos de genes que intervienen en la respuesta inmune hayan cambiado en el conejo después de la aparición de la mixomatosis y luego también después de la de la hemorragia vírica. Hay varios ejemplos de cambios en las frecuencias alélicas por selección cinegética, con sus correspondientes consecuencias adaptativas. Un trabajo clásico en genética cinegética descubrió que la caza selectiva de los mejores trofeos del carnero de las Rocosas provocó una disminución progresiva del peso corporal y el tamaño de la cuerna de los machos de una población en sucesivas generaciones (Coltman et al., 2003). Otro ejemplo clásico trata de la selección artificial del tamaño de la cuerna en machos de ciervo. Hay quienes eliminan los machos jóvenes con una cuerna poco desarrollada en un intento de conseguir una población de ciervos con mejores trofeos, matando para ello a los machos con peor fenotipo. Es el caso opuesto a la selección artifi- cial de los carneros de las Rocosas. Allí se matan los mayores machos adultos con el resultado de que los machos cada vez son más pequeños, y en este caso del ciervo se matan de muy jóvenes los machos de malas cuernas para obtener una población de machos con buenas cuernas. Se puede conseguir así, aunque la operación tiene sus riesgos. En Francia se descubrió que los alelos del gen que codifica la enzima mitocondrial isocitrato deshidrogenasa 2 (Idh-2) están relacionados con el tamaño de la cuerna en ciervos. En una población se mataron en años sucesivos los machos con poco desarrollo de la cuerna y el resultado fue un incremento en la población del alelo $I d h-2^{125}$ de ese gen, a la par que un incremento en el número de puntas de las cuernas en la población (Hartl, Lang, Klein y Willing, 1991; Hart et al., 1995). Sin embargo, unos británicos publicaron un trabajo (Pemberton et al., 1998) que describía que la supervivencia de las hembras jóvenes de ciervo era mayor cuando el gen $I d h-2$ estaba en heterocigosis, esto es, cuando en ambos cromosomas del par hay alelos diferentes de este gen. De modo que, en la población francesa, al eliminar machos con malas cuernas, eliminaban de la población uno de los alelos de este gen y con ello aumentaban la frecuencia en la que el gen $I d h$-2 es homocigoto y con ello disminuían a su vez la supervivencia de las hembras jóvenes. No es de extrañar que cuando se selecciona a favor o en contra de un fenotipo con base genética otros caracteres con base genética pueden verse afectados también. Hay variados casos de cambios de estructura genética provocados por la caza del tipo de cambios en el flujo genético, disminución de la diversidad genética y selección. No hay demasiados trabajos publicados dedicados al asunto, pero la evidencia que hemos encontrado hasta ahora nos sugiere que, en general, aunque la caza puede tener consecuencias genéticas en cuanto a flujo genético, disminución de diversidad y selección intencionada o no, estas consecuencias no suelen ser graves. De todos modos, faltan estudios y más aun monitorizando genéticamente poblaciones a lo largo de varias generaciones, que es como hay que hacerlo. Para hacer estos estudios basta con contar alelos para ver cambios genéticos en las poblaciones, interpretarlos e intentar aportar soluciones para la gestión cinegética, si es que son necesarias.

En la actualidad contar alelos es sencillo. El grado de conocimientos y tecnología que estamos consiguiendo en las últimas décadas es impresionante. Desde este punto de vista, son muy buenos tiempos para la ciencia que tenga que ver con la protección de la naturaleza y con la gestión de la caza. Corren tiempos emocionantes para la genética, la genómica, 
la proteómica y todas las “ómicas". En 1990 comenzó el Proyecto Genoma Humano, con el objetivo de secuenciar todo nuestro genoma. La secuenciación del genoma se completó en 2004 (International Human Genome Sequencing Consortium, 2004). Desde entonces se han secuenciado genomas enteros de muchas personas, de neardentales y de más de sesenta especies. No solo aumentan los conocimientos genéticos, sino que las técnicas para secuenciar y para estudiar marcadores genéticos avanzan enormemente a la vez que se abaratan sus costes en dinero y tiempo. Sin embargo, todos estos avances científicos útiles para la protección de la naturaleza están ocurriendo justo en nuestros días, a la vez que la naturaleza está sufriendo una crisis de extinciones de especies como nunca ha ocurrido en la historia del planeta. ¿Cómo es posible que todos estos conocimientos de aplicación inmediata a la protección de la naturaleza no sirvan para impedir su destrucción? La respuesta es porque los conocimientos no se aplican o, en el caso de las especies cinegéticas, se aplican mal.

\section{MALA APLICACIÓN DE LA GENÉTICA A LA GESTIÓN CINEGÉTICA}

"La tecnología es una forma de organizar el mundo de tal manera que el hombre no tenga que experimentarlo". Eso lo dijo Max Frisch. Esta frase creo que explica algunos casos de mala aplicación de la ciencia a la gestión cinegética. En ocasiones los conocimientos genéticos obtenidos con alguna tecnología se interpretan con elucubraciones abstractas equivocadas alejadas de la realidad. El divorcio que hay muchas veces entre el conocimiento científico y su aplicación empieza a veces desde el principio, desde la propia normativa que gobierna la caza. Hay dos tipos de errores en las normativas o en su aplicación: a) no regular una especie o subespecie cuando esa regulación es necesaria y b) regularlas cuando tal protección no es necesaria. Tanto una terminología imprecisa como unos conocimientos científicos insuficientes hacen que algunas decisiones que se toman en normativas y actuaciones sean incorrectas e indefendibles. Las consecuencias potenciales tanto de la sobreprotección como de la nula protección afectan directamente a la biodiversidad, al igual que lo hace indirectamente el desviar recursos hacia actuaciones innecesarias, cuando no perjudiciales. Luego los científicos nos podemos perder en elucubraciones abstractas equivocadas alejadas de la realidad y las administraciones encargadas de la normativa y su aplicación pueden perderse en ilusiones y eso en el mejor de los casos, que es cuando disponen de presupuesto para el manejo de las pobla- ciones cinegéticas. Se hará "la genética" del bicho en cuestión, esto es, se usará la tecnología para genotipar animales, pero con unos conceptos tan alejados de la realidad que la aplicación en la gestión será nula en el mejor de los casos y perjudicial las más de las veces. Es preferible ahorrarse el dinero y el esfuerzo.

Hay varios casos de mala aplicación de la genética a la gestión cinegética. El caso de la perdiz es un ejemplo paradigmático de mala gestión y es sobre el que me voy a extender más porque en esta especie se da casi todo el catálogo de malas aplicaciones de la genética en la gestión cinegética.

\section{EL CASO DE LA PERDIZ ROJA}

Hemos dicho que en poblaciones cinegéticas puede haber problemas de pérdida de diversidad por interrupción del flujo genético, por fragmentación o aislamiento de poblaciones o por caza excesiva o cualquier otra causa que provoque un cuello de botella. También puede haber alteraciones genéticas debidas a selección. Esos son problemas genéticos de lujo. No se estudian pérdidas de diversidad genética ni selección más allá de un puñado de trabajos académicos que no se aplican. No se les dedica la debida atención ni presupuesto aunque, con diferencia, el problema genético principal de la caza es otro.

Este artículo comienza con un párrafo que Miguel Delibes escribió en 1964. Se refería a que por entonces todavía quedaban animales en España, aunque en otros países más desarrollados ya no quedaban suficientes como para cazarlos, así que los extranjeros ponían los animales, esto es, que los criaban en granjas y los soltaban en el campo para cazarlos. Miguel Delibes se refería a la perdiz. A nadie se le escapa que un animal nacido y criado en una granja es, por definición, ganado o ave de corral. Así que los extranjeros cazaban ganado cinegético en sus países y para cazar animales silvestres tenían que venir a España. El tractor y la cosechadora llegaron a España y desde hace décadas ya la perdiz y otras especies "se ponen" en el campo aquí también. Por supuesto, Delibes previó lo que iba a pasar, pero creo que ni en sus peores pesadillas llegó a imaginarse lo mal que se iban a poner los animales en el campo. Medio siglo después de las palabras de Miguel Delibes, un hijo y un nieto suyos describen la suelta de cerdos vietnamitas, un animal exótico que está creando poblaciones asilvestradas en España y que hibrida con nuestro jabalí (DelibesMateos y Delibes, 2014). No es ya que se suelten animales, es que se puede soltar cualquier cosa y de cualquier manera. 
Las ideas que tenemos de la perdiz, como de otras especies cinegéticas, están llenas de ignorancias, prejuicios, lugares comunes, medias verdades e intereses. Unas décadas atrás algunos cazadores dieron la voz de alarma porque algunas perdices resultaban malas para el lance cinegético al no comportarse como lo habían hecho siempre las perdices silvestres. Las encontraban mansas. En el año 2000 se decía que esta mansedumbre se debía a la suelta de perdices de granja, pero no perdices rojas (Alectoris rufa) autóctonas, sino híbridos entre perdiz roja y perdiz griega (Alectoris graeca). El motivo para forzar esa hibridación en cautividad era, decían, porque la perdiz griega pone más huevos, con lo que la producción de la granja aumenta, y con ella el beneficio económico. La suelta de híbridos está prohibida por ley así que, aunque solo fuera por eso, es un asunto de interés y se hicieron y publicaron trabajos científicos que daban fe de esta hibridación entre perdiz roja y griega y se describían incluso marcadores genéticos para detectar la hibridación. Lo primero que llama la atención es que se diga sin más que la perdiz griega pone más huevos que la roja. Hay guías de aves que dan la información de las puestas. Consultando la primera a mano vemos que la puesta de la perdiz griega es de ocho a catorce huevos, que no es mayor que la de la perdiz roja (del Hoyo, Elliott y Sargatal, 1994). Averiguar si la perdiz roja se hibrida con griegas o con lo que sea es muy sencillo, rápido y barato. Basta secuenciar un segmento de ADN mitocondrial de varias perdices criadas en granjas cinegéticas y comprobar si las secuencias son propias de perdiz roja, de griega o de qué. El ADN mitocondrial se hereda por vía materna, así que algunas perdices híbridas tendrán ADN mitocondrial propio de perdiz roja, pero otras híbridas habrán heredado el ADN mitocondrial extraño por vía materna. Ni siquiera es necesario secuenciar, ya que es posible técnicamente conocer a qué especie pertenece un segmento de ADN mitocondrial de un modo indirecto que es aún más rápido y económico que la secuenciación (Blanco-Aguiar et al., 2008). Eso se hizo y no se encontró que en España la perdiz roja se hubiese hibridado artificialmente con la griega, pero sí con otra especie, la perdiz chúkar (Alectoris chukar), y de qué manera. La hibridación es generalizada en granjas cinegéticas y cada vez más frecuente en el campo. Donde dije digo, digo Diego. Sin más, se explicó esta práctica de hibridación artificial porque la perdiz chúkar pone más huevos. Comprobando esta afirmación en la misma guía de aves leemos, de nuevo, que no tiene mayor puesta. En realidad, las perdices rojas se han cruzado en granjas cinegéticas con líneas de perdices chúkar domesticadas que han sido seleccionadas para mansedumbre, para que críen mejor en una jaula y para mayor producción de huevos. No se han hibridado las patirrojas con chúkar silvestres, sino con aves domésticas de corral. Se han creado así líneas de perdices domesticadas híbridas entre perdices roja y chúkar y estos híbridos son los que se crían en granjas cinegéticas y se sueltan en toda el área de distribución de la perdiz roja. También se han creado artificialmente híbridos entre perdiz chúkar doméstica y perdiz griega para soltarlos en los países del área natural de distribución de la perdiz griega. No acabaron aquí los equívocos. Hace una década ya se sabía que estudiando de una forma rápida y barata el ADN mitocondrial de las perdices podíamos averiguar si en las poblaciones silvestres y granjas había híbridos. Sin embargo, el ADN mitocondrial se hereda por vía materna, así que los machos híbridos o aun machos de perdiz chúkar pura no transmiten su ADN mitocondrial a la descendencia. El ADN mitocondrial tiene sus limitaciones y se planteó la necesidad de acudir a los cromosomas para conseguir marcadores genéticos que diagnosticasen hibridación y que se heredasen de ambos padres. Esos marcadores genéticos son secuencias de ADN que son siempre de una manera en perdices rojas y siempre de otra en perdices chúkar, de modo que, si encontramos una secuencia propia de chúkar en una perdiz roja, tenemos la evidencia de que esa perdiz roja proviene de la hibridación con una perdiz chúkar. Además, debido a la forma en que se habían creado los híbridos, se necesitaba encontrar varios marcadores genéticos, cuantos más mejor.

Los híbridos de perdices roja y chúkar se han construido por retrocruzamientos de los híbridos de primera generación con perdiz roja. Voy a explicarlo. Un híbrido entre una perdiz roja y otra chúkar tiene la mitad de su genoma de perdiz roja y la otra mitad de chúkar. Esto es así porque ha recibido un juego de cromosomas del padre y el otro juego de la madre. En realidad, somos un poco más de nuestra madre que de nuestro padre porque heredamos de ella un poco más de genoma, que es el ADN mitocondrial. Los híbridos de primera generación son morfológicamente intermedios entre ambas especies y se reconocen a simple vista. No son estos híbridos los que se sueltan. Los híbridos de primera generación se cruzaron con perdices rojas, eso es el retrocruzamiento. Estos híbridos del primer retrocruzamiento tienen una cuarta parte de su genoma propio de perdiz chúkar y son morfológicamente indistinguibles de perdices rojas. Un segundo retrocruzamiento daría híbridos con un $12,5 \%$ de genoma de chúkar. No sabemos cuán- 
tos retrocruzamientos se hicieron. Sabemos que se hizo en Francia. En cualquier caso, en los sucesivos retrocruzamientos se seleccionaron aquellas perdices híbridas que conservaban las características de las perdices chúkar domésticas que son deseables para los criadores de perdices. No basta con uno o dos marcadores genéticos cromosómicos. En cada retrocruzamiento el porcentaje de genoma de perdiz chúkar del híbrido se reduce a la mitad, de modo que algunos marcadores genéticos propios de chúkar no van a pasar a la descendencia. A más marcadores genéticos, más posible es seguirle la pista a la existencia de hibridación en retrocruzamientos sucesivos. El límite en el número de marcadores que hay que emplear está en la cantidad de lugares diagnósticos de hibridación que seamos capaces de encontrar en el genoma y en el dinero que cuesta estudiar un número elevado de marcadores con la tecnología actual. Se ha conseguido una buena colección de marcadores genéticos para hibridación que, sin embargo, se están aplicando mal. Una vez conseguido un buen número de estos marcadores genéticos cromosómicos surgieron voces que opinaban que no era necesaria su aplicación. El argumento era que ninguna perdiz de suelta llega a sobrevivir en el campo, por lo que no suponen un peligro para la integridad genética de las poblaciones silvestres de perdiz roja. Este argumento no era más que un deseo. Se comprobó pronto que algunas perdices de suelta llegan a sobrevivir y reproducirse en libertad, contaminando genéticamente a la perdiz roja silvestre. No se sabe con precisión cuántas perdices se sueltan en España cada año. Las estimas varían entre unos tres y unos seis millones de aves. No se conoce tampoco con precisión qué porcentaje de perdices soltadas llega a sobrevivir y reproducirse. El porcentaje dependerá de cada lugar y las circunstancias y las estimas que se conocen son muy variadas. Supongamos que anualmente se sueltan en España cuatro millones de perdices de las que el $5 \%$ llega a reproducirse al menos en la siguiente temporada de cría. Eso significaría que cada año se están soltando en el campo 200.000 perdices híbridas que llegan a sobrevivir como para cruzarse entre ellas y con las perdices rojas silvestres para dar lugar a una descendencia de híbridos. Esas 200.000 perdices se multiplican por los hijos que tienen y esa es la magnitud anual de la destrucción de la integridad genética de la perdiz roja. Cada año la extensión de la hibridación aumenta, tanto en el grado de hibridación de los animales como en la extensión geográfica donde se da la hibridación. Por un lado, cada vez se sueltan perdices de granja en más lugares y, por otro, las perdices se mueven, extendiendo geográficamente el rango que ocupan las poblaciones híbridas. Cabe esperar que exista selección natural en contra de algunas variantes genéticas de los híbridos que causen una menor supervivencia en libertad, es posible, pero poco puede hacer la selección natural contra la selección artificial de imponer a la naturaleza millones de genomas domésticos cada año. Era urgente aplicar los marcadores genéticos diagnósticos de hibridación para intentar pararla. Así se hizo y se sigue haciendo, pero se aplican mal. Los marcadores genéticos se están usando mal porque se aplican a cada perdiz individualmente en lugar de tomar la población como unidad de estudio, con el resultado de que perdices en las que no se ha encontrado evidencia de hibridación son consideradas como perdices rojas puras cuando, por lógica, son híbridas. Además, se puede demostrar. Voy a intentar explicarlo.

Pongamos por caso que se dispone de veinte marcadores genéticos diagnósticos para detectar hibridación. Si en una perdiz roja se encuentra al menos un marcador genético exclusivo de perdiz chúkar, se puede afirmar sin lugar a duda que esa perdiz, en su genealogía, se ha hibridado y no es genéticamente pura. Sin embargo, veinte marcadores genéticos son veinte lugares en el genoma, y el número de sitios en el genoma en los que el ADN de una perdiz híbrida puede potencialmente contener una base de perdiz chúkar es astronómico. La hibridación puede estar en muchos millones de lugares del genoma, pero miramos solo en veinte. En cada retrocruzamiento sucesivo se pierde la mitad de genoma de chúkar y es posible que en los retrocruzamientos de una perdiz se hayan perdido esos veinte lugares que podemos estudiar pero que permanezcan otros millones de lugares con genoma de chúkar. Esa perdiz será híbrida, pero indetectable con esos veinte marcadores. Sin embargo, si se genotipase esa perdiz con marcadores diagnósticos adicionales sí sería posible demostrar su naturaleza híbrida, porque se estarían mirando otros sitios del genoma que sí podrían haberse conservado desde chúkar en los retrocruzamientos. ¿Cuántos marcadores genéticos hay que usar entonces? La respuesta es que no es un asunto importante, aunque se han hecho algunos cálculos matemáticos (Boecklen y Howard, 1997; Kanda, Leary, Spruell y Allendorf, 2002) que demuestran que hacen falta muchos marcadores para no cometer un error grande. Por ejemplo, con veinte marcadores diagnósticos la mitad de los híbridos de una población no se detectarían para porcentajes de hibridación del genoma similares a un quinto retrocruzamiento. Los resultados dan poco 
consuelo a quien quiera averiguar si un animal concreto es híbrido si no ha sido detectada introgresión. Si se dispusiera de cien marcadores diagnóstico el poder de detección de hibridación es ya suficiente para que, aun siendo imposible detectar a todos los híbridos, el error cometido sea pequeño. Un problema es que no disponemos de momento de tantos marcadores diagnóstico pero otro problema sería que, de tenerlos, habría que pagar el tiempo y el dinero que cuesta genotipar tantos loci, algo impracticable a los actuales costes para aplicarlo a la gestión cinegética. No obstante, el número de marcadores no es demasiado importante para su aplicación en gestión. Las pruebas genéticas para detectar hibridación se tienen que aplicar para la gestión cinegética no a animales individuales, sino a poblaciones. Si muestreamos una cantidad suficiente de perdices de una población, digamos sesenta, y estudiamos una docena o poco más de marcadores genéticos diagnósticos, si en esa población hay hibridación se detectará con seguridad la existencia de hibridación en el grupo. Si en esa muestra de perdices y utilizando los mismos marcadores no se encontrase hibridación, tendríamos la confianza de que la población es pura. Si se encontrase hibridación en un coto de caza no podríamos, en principio, saber cuántas perdices hibridaron, solo que en ese lugar hay hibridación. Lo que sí sabemos es que, aun en ausencia de más sueltas de híbridos, toda la población silvestre será híbrida en pocas generaciones, y todas ellas tendrán la misma proporción de contaminación genética: a esta población se la denomina "enjambre de híbridos". Si las sueltas de híbridos continúan, el grado de hibridación de cada perdiz silvestre irá aumentando generación tras generación. La población de perdices de una granja cinegética es un ejemplo de libro de enjambre de híbridos, ya que se han creado con ese fin. Si algunos animales de una granja dan positivo al test genético de la hibridación, todas las perdices de ese núcleo poblacional son híbridas. Esto lo da a entender también el hecho de que en aquellas granjas en las que se han eliminado perdices detectadas como híbridas, la producción de huevos sigue siendo tan elevada como antes, como corresponde a ponedoras hibridadas con perdices chúkar domésticas seleccionadas para producción.

Los marcadores genéticos hay que considerarlos en poblaciones naturales o artificiales pero se están aplicando a individuos, por eso se están utilizando mal. Algunas administraciones y particulares han buscado hibridación en los reproductores de sus granjas de perdices utilizando de diecisiete a veintidós marcadores genéticos. Cuando los tests genéticos de hibridación son positivos, lo que suelen hacer es eliminar aquellos individuos en los que se ha detectado hibridación, manteniéndose como reproductores los individuos en los que no se ha encontrado genoma de chúkar. Se están dando como puras esas perdices cuando, por lo expuesto hasta ahora, probablemente no lo sean. Se firman documentos que certifican que en una perdiz de granja "no hay evidencia de hibridación" y ese certificado hace legal al pájaro. Cualquiera sabe ya, pero creo que esta situación no puede sostenerse mucho tiempo, porque basta genotipar esas perdices con unos pocos marcadores adicionales para demostrar que son híbridas.

Todas las perdices de todas las granjas que he estudiado están hibridadas con perdiz chúkar doméstica. Con la ley en la mano, todas las granjas de perdices deberían cesar su actividad y quizá ser sancionadas. Eso no va a pasar porque aquí parece que cualquiera puede hacer mangas y capirotes de las leyes de caza. Quizá tampoco sea buena idea prohibir las sueltas sin más. Las perdices son importantísimas en los ecosistemas mediterráneos y no sabemos qué consecuencias podría tener dejar de soltar en el campo millones de presas para depredadores, algunos de ellos amenazados de extinción. Asumiendo que van a seguir las sueltas, al menos habría que procurar que las perdices de suelta que se crían en las granjas fueran rojas puras. Esto se puede conseguir con la ayuda de unos cuantos marcadores genéticos. La única manera de hacerlo es localizar poblaciones silvestres libres de hibridación y tomar de ellas pollos y huevos que se llevarían a una granja. Las perdices son aves prolíficas y en poco tiempo se obtendrían muchos reproductores libres de hibridación. El primer problema es dónde encontrar esas poblaciones libres de hibridación. Es obligado recordar que llevamos décadas mareando a esta perdiz. La extensión de la hibridación ha ido y va aumentando de tal manera que en la actualidad no es fácil encontrar lugares donde no haya llegado. Yo solo conozco la Casa de Campo de Madrid, el Parque Nacional de Monfragüe, lugares recónditos de los Arribes del Duero y gran parte de Navarra. Puede haber más sitios, desde luego, o menos: en Navarra se prohibieron las sueltas de perdices, pero es posible que en el futuro se permitan, y en los alrededores de Monfragüe ya han aparecido híbridos. La situación es urgente porque es cuestión de tiempo que no quede ni una sola pareja de perdices rojas puras que podamos tener en un zoológico, aunque sea como muestra. La perdiz roja era una riqueza casi exclusiva de España que hemos despilfarrado. Lo que se caza ahora no es perdiz roja, sino un sucedáneo. 
El caso de la perdiz es un ejemplo de mala aplicación genética a la gestión. Se utiliza la tecnología, se usa un lenguaje propio de la genética, pero de una manera tan alejada de la realidad que el resultado es perjudicial. Hay más ejemplos similares, pero ni se pueden citar todos ni mucho menos describirlos en detalle porque no acabaríamos, pero sí que merecen ser citados brevemente algunos. Hay dos subespecies de conejo, Oryctolagus cuniculus cuniculus y Oryctolagus cuniculus algirus, la primera distribuyéndose al norte de la segunda, con una franja intermedia de hibridación natural. Se realizan sueltas sin control genético alguno, de manera que se sueltan conejos de una subespecie en el área de distribución de la otra y, por si el viceversa no fuera suficiente, se sueltan además conejos domésticos. El resultado es una progresiva homogenización genética de la especie, difuminándose la diferencia entre ambas subespecies.

Se han descrito numerosas subespecies de ciervo, que varían según los autores. Tradicionalmente, en la Península Ibérica se reconocían dos subespecies atendiendo a medidas morfológicas: Cervus elaphus hispanicus (Hilzheimer, 1909) y Cervus elaphus bolivari (Cabrera, 1911), si bien esta última se cuestionaba. Sin embargo, varios estudios genéticos publicados en las últimas décadas han echado abajo la clasificación taxonómica tradicional del ciervo (Randi, Mucci, Claro-Hergueta, Bonnet y Douzery, 2001; Ludt, Schroeder, Rottmann y Kuehn, 2004; Meiri et al., 2013). Resumiendo, lo que tales estudios han demostrado es que lo que se consideraba una sola especie es en realidad un par de especies diferentes: el ciervo canadiense (Cervus canadensis), que se distribuye por América y el este de Asia, y el ciervo común (Cervus elaphus), que es de menor tamaño y ocupa el resto del Paleártico. Se identificaron tres subespecies de ciervo canadiense. En cuanto al ciervo común, los resultados de los estudios no apoyaban la existencia de numerosas subespecies, sino que solo identificaron cuatro líneas evolutivas diferentes: una en Europa Occidental, otra en los Balcanes, una tercera en Oriente Medio y una última en África. En otras palabras: según varios estudios filogenéticos, la subespecie descrita en España, Cervus elaphus hispanicus, no existe y los ciervos peninsulares son indistinguibles filogenéticamente de los ciervos de toda Europa Occidental, con la excepción de los balcánicos. En ausencia de base genética que diferencie los ciervos peninsulares de los europeos, no cabe hablar de pruebas genéticas para diferenciarlos. Aunque se han dedicado recursos para diferenciar genéticamente ciervos de la Península Ibérica de ciervos centroeuropeos (sobreprotección o mala protección), se han dedicado muy pocos para caracterizar especies e hibridación interespecífica (infraprotección). En otros países europeos se han descrito casos de hibridación artificial del ciervo común con otros cérvidos, en particular con ciervo canadiense y con ciervo sika (Cervus nippon), sin que en España se considere gran cosa estos posibles casos de hibridación, ni desde el punto de vista científico ni desde el administrativo. Desafortunadamente, en España se han realizado sueltas de ciervos de otras especies y subespecies y de sus híbridos y apenas se han podido detectar por no haberse realizado las pruebas genéticas oportunas.

La hibridación antropogénica es el principal problema genético de las especies cinegéticas y la lista de animales que la sufren es larga. Hay cuatro grupos evolutivos de jabalíes: europeos, italianos, de Oriente Próximo y asiáticos. En España se importan jabalíes para ser soltados, sin que sepamos de dónde ni cómo ni si tiene consecuencias genéticas porque no se ha estudiado. Sí sabemos que hay quienes hibridan jabalíes con cerdo doméstico para conseguir animales híbridos de gran tamaño. Aunque no es difícil detectar hibridación de jabalí y cerdo, no tenemos idea de la magnitud de estas prácticas de hibridación ni de sus consecuencias en la naturaleza porque prácticamente no se hace ningún control genético (Goedbloed et al., 2013). Existe evidencia de hibridación de codorniz común (Coturnix coturnix) con codorniz japonesa de corral (Coturnix japonica) y que esta hibridación se ha producido artificialmente en granjas cinegéticas. Una vez en el medio natural, los individuos híbridos podrían a su vez cruzarse con codorniz común (ver una reciente revisión sobre el tema en Puigcerver et al., 2013). Los efectos de la suelta de individuos híbridos pueden ser muy perjudiciales para la codorniz común, por ejemplo, porque los primeros suelen tener tendencias sedentarias. También se han soltado perdices pardillas de una subespecie ajena a la Península, sin que haya control genético alguno de estas actividades ilegales ni en codornices ni en perdices.

Hay más ejemplos de especies cinegéticas que se sueltan con consecuencias genéticas en las poblaciones silvestres. Como no hay animales, hay que ponerlos. Al menos, podrían ponerse bien. No es mi intención describir los conocimientos genéticos y la situación de todas las especies cinegéticas, una a una, sino contar la lista de malas aplicaciones de la genética en la gestión cinegética. La perdiz ha servido de principal ejemplo y lo dicho de ella se puede tener en cuenta para las demás especies. Los peces podrían haber sido otro buen 
ejemplo. Todas las malas prácticas cinegéticas con consecuencias genéticas en el campo ya las han sufrido los ríos previamente. Las especies piscícolas tienen problemas por desaparición de poblaciones y especies autóctonas, translocaciones entre cuencas con subespecies diferentes y suelta de especies exóticas invasoras y de híbridos de salmónidos. Existe una rica literatura de las consecuencias genéticas de las malas prácticas piscícolas que, aunque no ha podido evitar la contaminación genética de nuestros peces, sí sirve al menos de notario de lo que ha ocurrido. Los peces nos han dado conocimientos genéticos para evitar y controlar estos problemas, aunque en la práctica estos conocimientos se traducen en nada. Estamos arrasando los campos de la misma manera que hemos arrasado los ríos, sin que los conocimientos adquiridos sirvan siquiera como escarmiento.

No todo son malas actuaciones. Por ejemplo, en la Comunidad Autónoma del País Vasco se crían y sueltan liebres europeas (Lepus europaeus) de la propia línea evolutiva de la región y para ello realizan un genotipado sencillo y económico del ADN mitocondrial de los animales. No entiendo por qué en el resto de las especies cinegéticas, pudiendo hacer las cosas bien, o se hacen mal o no se hacen. No entiendo tampoco por qué, salvo excepciones, los animales soltados no se marcan. Una perdiz anillada informaría al cazador de que no es silvestre sino de suelta y, si la fecha de la anilla es de unos años atrás, le diría además que, aunque de suelta, esa perdiz mala no será cuando ha sobrevivido tanto. Si la perdiz fuera híbrida la anilla nos diría la granja origen de la hibridación, o quizá el origen de una enfermedad si la perdiz aparece enferma. No entiendo por qué ni siquiera se anillan los animales soltados, aunque sí sé que cuando alguien no quiere que se sepa algo es porque tiene algo que ocultar.

\section{EL FUTURO DE LA CAZA}

La caza va mal y además tiene mala prensa. No hace falta más que leerla para ver que todas las noticias referidas a ella son negativas y cuando los cazadores hablan es para quejarse de algo. Al igual que a otras industrias, quizá a la industria cinegética le ha llegado el momento de reconvertirse. Las prácticas cinegéticas han estado cambiando continuamente a lo largo de la historia y seguirán haciéndolo. Hay que intentar desarrollar buenas prácticas de caza que, al ser respetuosas con la naturaleza, permitan que la misma caza se pueda seguir realizando y pueda ser legitimada como sostenible y beneficiosa para el medio natural. No sé el futuro de la caza, pero sí el presente. Para mí, el paradigma de la caza en la actualidad son unas perdices sembradas que salen delante de unos hombres que se ponen las botas con perchas de espanto sin dar un palo al agua ni jugar el lance.

\section{BIBLIOGRAFÍA}

Blanco-Aguiar, J. A., González-Jara, P., Ferrero, M. E., Sánchez-Barbudo, I., Virgós, E., Villafuerte, R. y Dávila, J. A. (2008). Assessment of game restocking contributions to anthropogenic hybridization: the case of the Iberian red-legged partridge. Animal Conservation, 11 (6), pp. 535-545. https://doi.org/10.1111/ j.1469-1795.2008.00212.x

Boecklen W. J. y Howard, D. J. (1997). Genetic Analysis of Hybrid Zones: Numbers of Markers and Power of Resolution. Ecology, 78 (8), pp. 26112616. https://doi.org/10.1890/0012 9658(1997)078[2611:GAOHZN]2.0.CO;2

Butler, R. A. (2005, 16 de noviembre). World deforestation rates and forest cover statistics, 2000-2005. Mongabay. News and Inspiration from Nature's Frontline. [En línea]. Disponible en https://news.mongabay.com/2005/11/ world-deforestation-rates-and-forestcover-statistics-2000-2005/
Coltman, D. W., O’Donoghue, P., Jorgenson, J. T., Hogg, J. T., Stroceck, C. y FestaBianchet, M. (2003). Undesirable evolutionary consequences of trophy hunting. Nature, 426 (6967), pp. 655-658. https://doi.org/10.1038/nature02177

Delibes-Mateos, M. y Delibes, A. (2014). El cerdo vietnamita, nueva especie asilvestrada. Quercus, 335, pp. 68-69.

Funk, D. C. y Brown, M. E. (2009). Declining global per capita agricultural production and warming oceans threaten food security. Food Security: The Science, Sociology and Economics of Food Production and Access to Food, 1 (3), pp. 271-289. https://doi.org/10.1007/ s12571-009-0026-y

Goedbloed, D. J., Megens, H. J., van Hooft, P., Herrero-Medrano, J. M., Lutz, W., Alexandri, P., Crooijmans, R. P. M. A., Groenen, M., van Wieren, S. E., Ydenberg, R. C. y Prins, H. H. T. (2013). Genome-wide single nucleotide polymorphism analysis reveals recent genetic introgression from domestic pigs into Northwest European wild boar populations. Molecular Ecology, 22 (3), pp. 856-866. https://doi. org/10.1111/j.1365-294X.2012.05670.x

Gortázar, C., Che Amat, A. y O’Brien, D. J. (2015). Open questions and recent advances in the control of a multi-host infectious disease: animal tuberculosis. Mammal Review, 45 (3), pp. 160-175. https://doi.org/10.1111/mam.12042

Hartl, G. B., Klein, E., Willing, R., Apollonio, M. y Lang, G. (1995). Allozymes and the genetics of antler development in red deer (Cervus elaphus). Journal of Zoology, 237 (1), pp. 83-100. https:// doi.org/10.1111/j.1469-7998.1995. tb02748.x

Hartl, G. B., Lang, G., Klein, E. y Willing, R. (1991). Relationship between allozymes, heterozygosity and morpho- 
logical characters in red deer (Cervus elaphus), and the influence of selective hunting on allele frequency distribution. Heredity, 66, pp. 343-350. https:// doi.org/10.1038/hdy.1991.43

Hoyo, J. del, Elliott, A. y Sargatal, J. (1994). Handbook of the Birds of the World. Barcelona: Lynx Edicions.

International Human Genome Sequencing Consortium. (2004). Finishing the euchromatic sequence of the human genome. Nature, 431 (7011), pp. 931-945. https://doi.org/10.1038/nature03001

Kanda, N., Leary, R. F., Spruell, P. y Allendorf, F. W. (2002). Molecular genetic markers identifying hybridization between the Colorado River-greenback cutthroat trout complex and Yellowstone cutthroat trout or rainbow trout. Transactions of the American Fisheries Society, 131 (2), pp. 312-319. https://doi.org/10.1577/15488659(2002)131<0312:MGMIHB>2.0. $\mathrm{CO} ; 2$

Kurt, F. G., Hartl, B. y Voelk, E. (1993). Breeding strategies and genetic variation in European roe deer Capreolus capreolus populations. Acta Theriologica, 38 (Supplement 2), pp. 187-194. http://rcin.org. pl/Content/13290/BI002_2613_Cz-402_Acta-T38-Suppl2-187-194_o.pdf

Ludt, C. J., Schroeder, W., Rottmann, O., Kuehn, R. (2004). Mitochondrial DNA phylogeography of red deer (Cervus elaphus). Molecular Phylogenetics and Evo-
Iution, 31 (3), pp. 1064-1083. https:// doi.org/10.1016/j.ympev.2003.10.003

Malhi, Y., Roberts, J. T., Betts, R. A., Killeen, T. J., Li, W. y Nobre, C. A. (2008). Climate change, deforestation, and the fate of the Amazon. Science, 319 (5860), pp. 169-172. https://doi.org/10.1126/science.1146961

McGowan, P. J. K. (1994). Phasianidae (Pheasants and Partridges). En del Hoyo, J., Elliott, A. y Sargatal, J. Handbook of the Birds of the World (Volume 2. New World Vultures to Guineafowl). Barcelona: Lynx Edicions, pp. 434-552.

Meiri, M., Lister, A. M., Higham, T. F. G., Stewart, J. R., Straus, L. G., Obermaier, H., González Morales, M. R., MarínArroyo, A. B. y Barnes, I. (2013). Lateglacial recolonization and phylogeography of European red deer (Cervus elaphus L.). Molecular Ecology, 22, pp. 4711-4722. https://doi.org/10.1111/ mec. 12420

Pemberton, J. M., Albon, S. D., Guinness, F. E., Clutton-Brock, T. H. y Berry, R. J. (1998). Genetic variation and juvenile survival in red deer. Evolution, 42, pp. 921-934. https:// doi.org/10.1111/j.1558-5646.1988. tb02511.x

Puigcerver, M., Sánchez-Donoso, I., Vilà, C., Sardà-Palomera, F., Morales-Rodríguez, P. A., Caballero de la Calle, J. R., Rodríguez-Teijeiro, J. D. (2013). Hibridación entre la codorniz común (Coturnix coturnix) y la codorniz de granja: estado de un problema de conservación. Ecosistemas. Revista científica de ecología y medio ambiente, 22 (2), pp. 48-53. https://doi. org/10.7818/ECOS.2013.22-2.08

Randi, E., Mucci, N., Claro-Hergueta, F., Bonnet, A., Douzery, E. J. P. (2001). A mitocondrial DNA control region phylogeny of the Cervinae: speciation in Cervus and implications for conservation. Animal Conservation, 4 (1), pp. 1-11. https://doi.org/10.1017/ S1367943001001019

Terborgh, J. (1999). Requiem for Nature. Washington, DC: Island Press

\section{Otros recursos}

Estadística anual de caza. Tablas resumen 2005-2010. [En línea]. Disponible en http://www.magrama.gob.es/es/ biodiversidad/estadisticas/estadistica_anual_caza_resumen_2005_2010_ tcm7-263274.xls

FAOSTAT - Food and Agriculture Organization of the United Nations. Statistics Division. [En línea]. Disponible en http:// faostat3.fao.org/home/E

United Nations - Department of Economic and Social Affairs. Population Division. [En línea]. Disponible en http://www. un.org/esa/population/unpop.htm

World Bank Open Data. [En línea]. Disponible en http://data.worldbank.org/ 